\title{
Esperando a Godot: Revelando el sentido sinsentido en la posmodernidad
}

Waiting for Godot: Revealing the M eaninglessness of M eaning in Postmodernity

José J. Contreras

U niversidad de Los Andes, M érida, Venezuela

E-mail: joaquin@ula.ve

\section{INTRODUCCION}

E SPERAN D 0 a Godot de Samuel Beckett es estrenada en París en 1953. Q ui-

E zás el cal ificativo más apropiado para describir la sensación que deja la obra en la mayoría de los espectadores es el de "desconcertante". D os seres humanos, con apariencia de vagabundos, se encuentran a la orilla de un camino en un paisaje desolado esperando y esperando y esperando a un tal Godot ${ }^{1}$ que no termina de hacer presencia pero que aunque "hoy no vendrá mañana de seguro, sin falta, lo hará", 3 . Lo desconcertante de la obra no es que G odot nunca llegue sino que pareciese que nada ocurriese en un constante ciclo de repeticiones y repeticiones y repeticiones en las que la cotidianidad de los personajes va revelando su sinsentido.

Es interesante notar cómo la discusión académica en torno a la obra de Beckett, y específicamente en torno a Esperando a Godot, ha venido girando en losúltimostreinta años. Por ejemplo, si vemos referenciastales como Rabinovitz (1977) y Velissariou (1982) podremos notar que el énfasis en estos estudios se concentraba en mostrar cómo la obra desencubre los límites de la modernidad y con ello su pérdida de poder. Rabinovitz, por su parte, se enfoca en exponer

\footnotetext{
${ }^{1}$ En inglés "G odot" puede sonar a algo parecido a "D iosote" en castellano.

${ }^{2} L$ a traducción de Esperando a Godot que utilizaremos en el presente artículo es una realizada por H ernán López G aray, M iguel C respo Alvarado y José J. Contreras, la cual no ha sido publicada para el momento en que el presente artículo es escrito. Para solventar este inconveniente presentaremos las citas en castellano con una nota al pie de página en la cual colocaremos la cita en inglés y que podrá ser revisada por el lector.

${ }^{3}$ Acto I. "Vladimir: He said that Godot was sure to come tomorrow".
} 
cómo la verosimilitud de los "objetos" modernos se pierde ante la evidencia de que el tiempo y el espacio no son tan absolutos como se pensaban. Velissariou más bien muestra cómo el lenguaje en Esperando a Godot devela la fragmentación del lenguaje moderno tras la "ausencia absoluta de los absolutos".

Sin embargo, cuando revisamos estudios más recientes notamos queel énfasis en la discusión de la obra se ha desplazado a mostrar cómo ella devela las características de lo que se ha llamado la "posmodernidad". Veamos igualmente dos referencias que pueden ilustrar el punto. Salatino (2001) se concentra en exponer cómo la obra revela el desamparo del hombreposmoderno y que muestra la condición humana "descarnada", tal y como ella es y ha sido. Campos, de un modo distinto mas no contrario al anterior, se enfoca en mostrar cómo la obra describe elementos claves de la época posmoderna tales como la discontinuidad, la fragmentación, la contradicción, la permutación, la destemporalización, la designificación y la destotalización.

El presente artículo quisiese detenerse en el medio de ese desplazamiento. Es decir, en ese punto que, como bisagra, lleva de lo moderno a lo posmoderno. Porque, a fin de cuentas, lo posmoderno es aún moderno. Lo posmoderno acude y significa desde lo moderno. Incluso, en oportunidades, lo posmoderno más que ser "post-moderno" parece ser "en pos de lo moderno". Y Esperando a Godot revela esto de una manera que sólo puede hacerlo una obra de arte excelsa.

En este orden de ideas el presente artículo seguirá un camino de indagación que buscará ir mostrando cómo los personajes de la obra manifiestan características que pueden ser celebradas a la luz de la modernidad y cómo, al mismo tiempo, estas características ya no son modernas y en este sentido revelan el "desamparo" - para usar el término heideggeriano de Salatino- del hombrecontemporáneo en la tragedia de su existencia.

\section{LA MODERN ID AD EN ESPERANDO A GODOT}

Q uizás el mejor modo de acercarnos a revelar la modernidad en la obra es a través de sus personajes. Ellos nos irán mostrando por qué la obra parece fundarse en las ideas fundamentales modernas y en sus estructuras sociales. Empe cemos, pues, la indagación a través de V ladimiro.

\subsection{Vladimiro moderno}

Para caracterizar a la modernidad, y especial mente cómo ella parece manifestarse en Vladimiro, podemos empezar por uno de sus impulsos más importantes 
que fue la duda sistemática expuesta por D escartes. D escartes, movido por un impulso vital de libertad, dudó de todo. D escartes dudó de la I glesia, de las concepciones tradicional es acerca del mundo, de lo que aparece y cómo lo hace. D escartes tuvo el coraje de dudar y hacer de la duda su modo de estar en el mundo. Con ello comenzó a sentar las bases para una nueva plataforma con carácter de verdad que entendiera la realidad.

En su dudar, D escartes logró darse cuenta de que si había algo de lo que no podía dudar era precisamente de que "estaba dudando". Por lo tanto, si estaba dudando entonces él estaba, era. D e este modo, el humano cartesiano es en su posibilidad de dudar y esta duda es lo que aparece allí como fundamental al pensamiento. Pero aún más, nótese que es gracias al pensamiento que D escartes puede asegurarse de su propia existencia. Por ello D escartes expuso su famosa tesis: cogito ergo sum, "pienso luego soy" y con ello inauguró una nueva concepción de la existencia humana, entendida ésta, fundamentalmente, como sustancia pensante.

Vladimiro, el personaje más importantedela obra, duda de manera sistemática, vital y comprometida, durante toda la obra. Esa duda vital le permite a V ladimiro realizar diversos razonamientos sobre variados temas que se le ocurren durante la espera del misterioso Godot. Por ejemplo, al inicio del Acto I, V ladimiro duda sobre una de las creencias religiosas cristianas más difundidas. Aquélla según la cual uno de los dos ladrones que fueron crucificadosal lado de Jesucristo fue salvado. Su duda se basa en la falta de razonamiento lógico de tal creencia. D elos cuatro evangelistas sólo uno menciona que un ladrón fue salvado; "de los otros tres, dos no mencionan a ningún ladrón y el tercero dice que amboslo insultaban"4. V ladimiro, de manera lógica, se pregunta por quécreerle al evangelista que menciona que uno de los dos ladrones fue salvado y no a los otros tres.

En esta duda en torno a una de las creencias cristianas se nos muestra con más claridad el carácter de la duda moderna. La modernidad se basa en la duda porque busca construir un piso firme que permita la verosimilitud, es decir, que lo real se considere de modo tal que sea verdadero. Pero, ¿por qué esa duda?, ¿por qué vivir dudando?, ¿qué sucede ante la real idad que se le presenta a D escartes que amerita dudar de todo? Pues bien, la duda sistemática cartesiana lo que pretende es posibilitar un piso que permita construir una verdad que se enfrente a la verdad revelada de la I glesia. $\mathrm{N}$ ótese el cambio fundamental quese lleva a cabo con el dudar moderno. El dominio de la Iglesia sobre la verdad pierde poder y es cuestionado. D e estemodo, el ser humano moderno secree en

${ }^{4}$ Acto I. "V ladimir: O ne out of four. Of the other three, two don't mention any thieves at all and the third says that both of them abused him". 
la capacidad de conseguir, por sí mismo, los basamentos de verdad que soporten su acción ante el mundo y ello puede hacerlo mediante el uso del pensamiento. Es en este sentido queel hombremoderno se sienteen un proceso deliberación.

Este cambio fundamental posibilita un desplazamiento en el modo de entenderse lo humano. En la Edad M edia los seres humanos se entendían como creaciones divinas, que eran herederos de un pecado original pero que habían sido redimidos por Jesucristo y por ello podían alcanzar la sal vación después de su muerte si sus acciones eran buenas. En la modernidad, de manera distinta, los seres humanos creen que fundamentalmente son seres pensantes. El otro ya no aparece como una creación divina sino como un ser pensante. En tanto que ser pensante, es capaz de decidir racionalmente qué hacer y en ello es capaz de actuar moralmente. Por lo tanto la dignidad humana es derivada de la capacidad de razonar y todo ser humano es digno porque es racional.

$D$ ado lo anterior se entiende por qué V ladimiro reacciona violentamente ante el trato que realiza Pozzo sobre Lucky: "tratar a un hombre... aś... C reo que... N o... Es un ser humano... ¡Q ué vergüenza!"5. El tratamiento bestial de Pozzo a Lucky mancilla su dignidad como ser humano. Por eso V ladimiro censura el comportamiento de Pozzo y aboga por el tratamiento digno del que es merecedor Lucky por el simple hecho de ser un hombre.

Vemos así cómo en V ladimiro parecen manifestarse principios fundamentales del hombre moderno. Por una parte encontramos la duda ante las creencias reveladas dela I glesia y por otra parte vemos su indignación ante el tratamiento humillante del que es objeto Lucky. Pasemos a revisar el caso de otro personaje de la obra: Pozzo.

\subsection{Pozzo moderno}

$D$ ado lo anterior entonces Pozzo no debeser un personaje moderno. Sin embargo el camino social que sigue la modernidad se al eja un poco del filosófico. En las ciudades europeas de la época renacentista va cuajando, poco a poco, un proceso de revolución política y económica que cambia la faz del mundo entero. La nobleza y las monarquías europeas, conformadas y justificadas dentro del marco religioso feudal, van perdiendo legitimidad. La riqueza deja de medirse en oro y piedras preciosas y empieza a girar en torno a los negocios que se realizan en losburgos. Empieza así a surgir con fuerza la burguesía como grupo de poder que socava la influencia de la nobleza tradicional.

${ }^{5}$ Acto I. "V ladimir: ... To treat a man... like that... I think that... no... a human being ... no... it's a scandal!" 
Bajo este nuevo esquema, la relación social tienelugar al rededor de losnegocios y del librejuego del capital. En este sentido, la burguesía concibe que en el espacio delo público lo que se debe procurar esel cuidado dela propiedad privada y la riqueza de los propietarios. Lejos va quedando la sociedad feudal de nobles y plebeyos para dar paso a una sociedad que se define en términos del capital.

D e este modo se crearon las condiciones de posibilidad para el surgimiento dela sociedad industrial y con ella de la distinción social básica entre propietarios y proletarios. Los primeros dueños de la propiedad y los segundos marginales a ella y asalariados. Claro que, idealmente, todos los seres humanos tendrían la posibilidad de adquirir propiedades y ellas le permitirían al canzar un cierto nivel deautonomía material individual para participar en igual dad de condiciones. Sin embargo, con el devenir del capitalismo empezó a hacerse palpable la profundización de la brecha entre aquellos que podían adquirir y acumular propiedades y los que no y que por ello quedaban marginales al orden social.

Y allí aparece con toda claridad Pozzo: el propietario. Pozzo entiende su papel. El sabeque si bien todo el discurso moderno de la igualdad de los hombres es importante, el propietario se encuentra en un nivel social superior. De hecho, Pozzo no cree que domina a Lucky ilegítimamente. Lo que ocurre es que Lucky ha renunciado a su derecho de "ponerse más cómodo" 6 y ello lo hace porque, según Pozzo, Lucky intenta causarle una buena impresión. Lucky realiza su función de la mejor manera para que Pozzo siga utilizando sus servicios. Para V ladimiro, Pozzo veja a Lucky, pero para Pozzo, Lucky escogió libremente (a derecho) llevar la vida quelleva como su maletero y sirviente para así obtener beneficios tales como las sobras de hueso de pollo de su patrón.

Sin embargo, Pozzo necesita justificar racionalmente su posición como propietario y ello lo hace siguiendo un razonamiento lógico del tipo moderno. En ello se muestra una profunda diferencia entre el tipo de razonamiento de Pozzo y el deV ladimiro. A Pozzo no lo muevela duda cartesiana quemuevea V ladimiro. Vladimiro, al dudar, piensa y busca verdad. Pozzo no. Pozzo sigue ciertas normas básicas de razonamiento que lo que buscan es o bien justificar su relación con Lucky o bien demostrar sus dotes discursivos "superiores" ante V ladimiro y Estragón como ocurre en el caso de la cháchara sobreel "misterio delos crepúsculos"7. El razonamiento deV ladimiro procura hallar verdad; el dePozzo inten-

\footnotetext{
${ }^{6}$ Acto I. "Pozzo: ... W hy he doesn't make himself comfortable? Let's try and get this clear. $\mathrm{H}$ ashenot the right to? C ertainly hehas. It follows that he doesn't want to... And why doesn't he want to? (Pause) G entlemen, the reason is this... He wants to impress me, so that I'll keep him".

${ }^{7}$ Se refiere al momento en el Acto I en que Pozzo da una seudo-disertación que comienza asi: "W hat is there so extraordinary about it? Q ua sky. It is pale and luminous like any sky at this hour of the day..."
} 
ta justificar sus intereses particulares. ¿Puede verse la profunda diferencia entre uno y otro? En V ladimiro la acción deriva de la verdad, mientras que en Pozzo la verdad deriva de sus intereses burgueses. La diferencia es sutil pero, al mismo tiempo, abismal.

Se hace notorio en esta sección que no es posible hablar de Pozzo sin hablar de Lucky y viceversa. Pozzo puede distinguirse como propietario porque existe un Lucky que es proletario. Pasemos entonces a revisar la modernidad en Lucky.

\subsection{Lucky... moderno}

H emos dicho que Pozzo es un personaje moderno y que éste no puede ser sin su Lucky. En consecuencia, Lucky también debe ser un personajemoderno. Ahora bien, Lucky contrasta en mucho con el hombre moderno del cual hablábamos cuando caracterizábamos a V ladimiro. ¿C ómo podemos hablar de Lucky como un personaje moderno si precisamente él es la oposición al hombre libre?

El trabajo es la acción vital del hombre que le permite construir un mundo artificial. El hombre, a diferencia de cualquier otro animal, fabrica un mundo deartificios dentro del cual procura tanto protegerse de las amenazas delo natural como dedicarse a las actividades que considera más suyas. Nótese que no estamos hablando aquí del laborar natural propio que le permite al hombre obtener su alimentación y vivienda y que es también característico de otras especies. Estamos refiriéndonos a las actividades humanas que hacen al mundo más humano.

A hora bien, en la modernidad el trabajo se enajena. El trabajo ya no le permite al hombre particular hacer mundo en el producto de su quehacer. La división del trabajo industrial hace que los hombres se constituyan en apéndices de grandes maquinarias de ensamblaje. EI hombre-máquina poco o nada tiene que ver con el producto final de la línea de ensamblaje. El producto final ya no es producto del hombre sino de la gran maquinaria industrial.

D e este modo encontramos que en la sociedad industrial, la época de los grandes inventos y de los descomunales avances tecnológicos, el trabajo enajena a los hombres de la construcción de su mundo artificial. El mundo se hace extraño al hombre, el mundo se enajena del hombre, ergo, el hombre se enaje na. H eahí Lucky... Lucky es el hombrequeya no esporquele ha sido enajenado el mundo que lo hace hombre.

$N$ ótese el giro que hemos dado, en la modernidad lo que hace ser al hombre es su pensar. Pensamiento que lo hace libre porque lo libera de las creencias tradicionales inverosímiles y en ello le permite construir su mundo humano a través del trabajo. Pero, con el trabajo industrial se enajena al hombre de su 
mundo humano. No es que en la sociedad industrial el hombre se liberó de la natural eza sino que el hombre se enajenó en cuanto ser humano.

El paisaje desterrado de Esperando a G odot en el cual "no hay nada"8 es muestra de ese mundo "humano" enajenado. El mundo des-terrado que separado de la naturaleza no llegó a ser un mundo más humano sino un camino de tierra y piedras, con un árbol seco, un chiquero y un montículo.

Es por ello que en la obra pareciese que nada sucediese, "no hay nada que hacer" ${ }^{\prime \prime}$, repiten Vladimiro y Estragón de cuando en cuando. N o sucede nada porque el mundo desterrado, el no-mundo, impide la ocurrencia. Todo lo que ocurre ocurre desde un marco más o menos establequelesirve defondo y quees el mundo. El mundo no aparece pero está allí como escenario que posibilita que en cada escena ocurran situaciones con sentido. Por ello, el mundo enajenado de la obra, ese mundo al que se le ha enajenado su condición de mundo, al no estar como fondo impide la ocurrencia y por ello todo queda en un eterno ciclo que se repite, se repite y se repite recurrentemente ${ }^{10}$.

H emos visto queV ladimiro, Pozzo y hasta Lucky son exponentes que muestran la sociedad moderna desde diversas facetas. Veamos ahora el caso deEstragón.

\subsection{Estragón, ¿moderno?}

Estragón es el personaje más complicado de la obra. N o hace nada, sólo duerme. Se come uno que otro tubérculo que le da V ladimiro o algún hueso que le deja Pozzo. M endiga, se burla de todos y sabotea los razonamientos... Seaburre, simplemente. Estragón sabe muy bien que para él "no hay nada que hacer"...

¿Q uién es Estragón?... Un poeta, "¿que no es obvio?"11 o, mejor dicho, lo fue. H emos estado refiriéndonos a la modernidad, su duda sistemática y su concepción según la cual lo que hace al hombre hombre es su pensamiento. Ahora bien, cuando decimos pensar y razonar en la modernidad nos estamos refiriendo a un razonamiento del tipo lógico-matemático.

El pensamiento moderno procura aplicar la lógica matemática a todo pensa-

${ }^{8}$ Acto II. "Pozzo: W hat is it like? / V ladimir: (looking round). It's indescribable. It's like nothing. There's nothing".

9 "N othing to be done".

${ }^{10}$ Arendt (1958) nos dice lo siguiente: "W ithout a world into which men are born and from which they die, there would be nothing but changeless eternal recurrence, the deathless everlastingness of the human as of all other animal species" (p. 97).

${ }^{11}$ Acto I. "Vladimir: You should have been a poet / Estragón: I was (Gesture towards his rags) Isn't that obvious?". 
miento para que pueda ser considerado como cierto. D e modo similar a la matemática, el pensamiento moderno comienza desde principios axiomáticos incuestionables para que a partir de allí, y mediante la aplicación de estrictas reglas lógicas, se pueda construir un razonamiento que sea válido.

Con ello el pensamiento moderno, en su reducción a lo lógico-matemático, elimina la posibilidad de toda otra manifestación de pensamiento que no pueda ser encasillada dentro de las férreas reglas de la lógica deductiva e inductiva. H e ahí Estragón. La poesía no tiene cabida en la modernidad. Estragón no tiene "nada que hacer". N ada de nada.

Es por eso que Estragón sólo parece tomarse en serio ese pequeño momento poético - quelogró colarse por al guna de esas rendijas que quedan en las grandes represas- en el que ante la excusa de no pensar se revelaron las voces muertas en su significación de la designificación, hablando al unísono y sonando como alas porque haber vivido no les fue suficiente y haber muerto no les basta... Es en este momento que Estragón intenta rebasar las fronteras reducidas del pensamiento lógico moderno para regresar resuel tamente a la naturaleza. Sin embargo, V ladimiro le recuerda que eso ya lo han intentado fallidamente. Por eso lo peor no era el intento de retorno a la naturaleza, lo peor era haber pensado que el único modo verdadero de pensar era el razonamiento lógico-matemático... "Eso es obvio", dice Estragón...

"Silencio..."

En este silencio abismal V ladimiro se encuentra ante la limitación moderna y allí Estragón cambia de lengua y pregunta: “¿Q uevoulez-vous?". V ladimiro lo medita y responde: "Exactamente"... "N o estuvo mal como veta a explorar para matar el tiempo" 12, responde Estragón... ¿Q ué ha sucedido aquí?

En el largo silencio queda suspendida la lid entre poesía y razón. Esta suspensión no responde a la imposibilidad de victoria de una u otra, ni tampoco a un espacio común de resolución. La suspensión responde a la desaparición del piso sobre el cual los contrincantes estaban. Estragón parece saberlo y pregunta acuciosamente "Q ue voulez-vous?". Vladimiro sólo puede responder con una aserción sin posibilidad de solución.

La vida humana es una constante proyección sobre el incierto futuro. Proyección que se da con un cierto sentido de búsqueda. Búsqueda que procura

${ }^{12}$ Acto II. "Estragon: We should turn resolutely towards N ature. / V ladimir: We've tried that. / Estragon: True. / V ladimir: O h it's not the worst, I know. / Estragon: W hat? / V ladimir: To have thought. / Estragon: O bviously. / Vladimir: But we could have done without it. I Estragon: Q ue voulez-vous? / V ladimir: I beg your pardon? / Estragon: Q ue voulez-vous. I Vladimir: Ah! que voulez-vous. Exactly. / Silence. Estragon:

That wasn't such a bad little canter". 
lograr un bien. Logro que no se consuma como cuando se alcanza un objetivo, sino que se va realizando, se va haciendo realidad, se va haciendo real. La constante proyección de la vida humana va buscando la realización de lo bueno.

Pero logro de qué bien, ¿de cualquier bien?... N o. Se trata de un bien trascendental que brinde sentido a la vida. En la época moderna setrataba del logro de la liberación. Liberación que se soportaba sobre un piso que era la constante y progresiva autonomía en el uso de la razón por parte de todos los seres racionales en contraposición al tutelaje ejercido por la verdad revelada de la I glesia. Pero, ¿es eso lo que quiere V ladimiro? ¿V ladimiro está liberándose de la verdad revelada?... "Q ue voulez-vous?"

¡N o!, claro que no, Vladimiro está esperando a Godot...

\section{ESPERAN D O A GODOT}

El afán de libertad moderno no es lo que vitaliza a Vladimiro y por lo tanto él no es propiamente un personajemoderno. En realidad, ninguno delos personajes de la obra está movido por tal afán, en consecuencia, ninguno de los personajes es propiamente moderno. Pero, zacaso no hemos mostrado cómo cada uno de ellos presenta características fundamental es del hombremoderno?, ¿cómo es posible que ahora digamos que no son modernos?

El hacer de V ladimiro está condicionado por la espera de G odot. La espera de G odot se encuentra como principio vital de V ladimiro. Es esa espera la que brinda sentido a todo lo que hace (y deja de hacer) Vladimiro a lo largo de la obra. Por ello, él no duda para construir un nuevo piso de verdad, ni defiende a Lucky en tanto que ser humano con capacidad de moralidad, sino que hace todo lo que hace para que el tiempo pase mientras espera.

Vladimiro lo dice claramente en un momento en que medita si ayuda a Pozzo que yace en el suelo ciego:

Q uéestamos haciendo aquí, ése es el problema. Y si en algo hemos sido bienaventurados es en el hecho de que sabemos la respuesta. Sí, en medio de esta inmensa confusión, sólo una cosa es clara: Estamos esperando la venida de Godot ${ }^{13}$

Para luego rematar diciendo:

${ }^{13}$ Acto II. "V ladimir: ... W hat are we doing here, that is the question. And we are blessed in this, that we happen to know the answer. Yes, in this immense confusion one thing alone is clear. We are waiting for Godot to come". 
Admitamos francamentequeel hastío seapodera de nosotros avasalladoramente, de eso no hay duda. ¿Y qué hacemos cuando al fin un entretenimiento toca a nuestras puertas?... Incomprensiblemente lo desaprovechamos. Ven, ipongámonos a trabajar! ${ }^{14}$

Para entretenerse, para hacer algo y no hastiarseen demasía. V ladimiro duda, Vladimiro reprocha, Vladimiro ayuda, Vladimiro hace todo lo que hace para "matar el tiempo". Por ello, también se entretiene con el baile y la disertación de Lucky. Esperando a G odot transcurre su vida y su quehacer.

Sin embargo, V ladimiro sabe que en todo ello hay un serio problema. Q ue su hacer procure "matar el tiempo", que el hastío se apodere avasalladoramente de su devenir, es síntoma, quizás indudable, de que el mundo ha desfallecido ante una espera prolongadamente insatiffecha. Por ello, V ladimiro sabe que ante estas condiciones en las que el mundo desmaya "las horas se hacen más largas, y nos fuerzan a engañarlas con procedimientos que - cómo decirlo- a primera vista pueden parecer razonables, hasta que se nos convierten en hábitos. Claro, uno puede decir que esto es así para prevenir quela razón colapse, sin lugar a dudas. Pero, zacaso la razón no ha estado desde hace ya largo tiempo sumiéndose gradual mente en la noche sin fin de las profundidades abismales? Esto es lo que a veces no deja de causarme asombro. ¿Sigues mi razonamiento?"15.

Vladimiro no sabe exactamente qué pasa pero sabe que el mundo moderno desmaya. Estragón, el poeta, le responde: "todos nacemos con la razón perdida. $Y$ al gunos pasan toda su vida sin poder recuperarla"16. Pero V ladimiro no entiende la respuesta. Estragón no le ha respondido que todos nacemos débiles mentales, lo que Estragón leha dicho es que la razón es sólo un modo de pensamiento de por sí reducido. Por ello, todos nacemos con la razón perdida -es decir, no la tenemos naturalmente- y no todos logran recuperarla - no todos logran confinarse a ella- como es en efecto el caso de Estragón.

De lo anterior se entiende que Vladimiro quizás más que moderno es posmoderno. Vladimiro vive en las creencias de la modernidad, pero en una época en que el afán de libertad que preñaba de sentido todo el quehacer mo-

${ }^{14}$ Acto II. "Vladimir: ... N 0, don't protest, we are bored to death, there's no denying it. G ood. A diversion comes along and what do we do? We let it go to waste. Come, let's get to work!".

${ }^{15}$ Acto II. "V ladimir: All I know is that the hours are long, under these conditions, and constrain us to beguilethem with proceedings which - how shall I say- which may at first sight seem reasonable, until they become a habit. You may say it is to prevent our reason from foundering. No doubt. But has it not long been straying in the night without end of the abyssal depths? That's what I sometimes wonder. You follow my reasoning?"

${ }^{16}$ Acto II. "Estragon: ... We are all born mad. Some remain so". 
derno ha desaparecido. V ladimiro vive en el mundo moderno de la razón, pero ya no en actitud de liberación, sino procurando atarse a un tal Godot.

Estragón, por su parte, si bien logra percatarse del reduccionismo moderno, no puede escapar a él. Estragón quiere irse, "lejos muy lejos"17, de donde están. Pero él sabe que la escapatoria no reside en irse por el camino que deambulan Pozzo y Lucky. Estragón no logra divisar escapatoria al guna. Sus intentos frustrados por volver resueltamente a la naturaleza y su imposibilidad por avizorar escapatoria le llevan a exasperarse, a burlarse, a rendirse y, en últimas, a intentar el suicidio como modo terminal. En resumidas cuentas, una vida sinsentido, una vida en la que el quehacer no hacenada, es una especie de muertesinfín ala que Estragón quiere hallar fin.

Pozzo y Lucky viven una suerte similar. Entre más se enajena Lucky, más se enajena Pozzo. En el Acto I, Pozzo nos muestra cómo va perdiendo velozmente sus cosas: su reloj, su vaporizador, su pipa. Pierde sus propiedades y él - Pozzoes un propietario... Pozzo va desapareciendo ante el desvanecimiento de la humanidad (y la aparición de la monstruosidad) de Lucky. En el Acto II Lucky, mudo mudo, yace inerte; Pozzo ciego, sin cesta y sin vino, sin pipa y sin vaporizador, no posee nada. "iPobre Pozzo!"18, dice V ladimiro.

Pozzo y Lucky, ánimas que deambulan atareadas en el limbo de la modernidad ya pasada.

¿Q uién es Godot? G odot al igual que D ios brinda sentido. En la obra G odot brinda sentido a todo el quehacer de nuestros personajes. Godot nunca aparece pero, al mismo tiempo, impregna con su presencia no-presente todo lo que hacen, hablan y piensan los personajes. En fin, G odot es condición que posibilita todo lo que sucede en Esperando a Godot. El es una especie de causa primera que permite que todo lo que aparezca en escena sea lo que es. La espera de G odot es la narrativa que teje el sentido del quehacer de V ladimiro y Estragón, de Pozzo y de Lucky.

N ótese que a G odot, en tanto que fuente de sentido, le está negado llegar, puesto que, como Dios, debe dominar la acción humana desde su no-llegar Ilegando ${ }^{19}$. Pero G odot, quien es quizás mejor descrito en el monologo de Lucky como ese dios personal que en su divina apatía, su divina atambía ${ }^{20}$ y su divina afasia, "nos ama mucho, con al gunas excepciones, no se sabe por qué"21 no logra

\footnotetext{
${ }^{17}$ Acto II. "Estragon: ... Let's go far away from here".

${ }^{18}$ Acto II. "Vladimir: Poor Pozzo!".

${ }^{19}$ Ver Butler (1991).

${ }^{20}$ A tambía: C arencia de la capacidad de emocionarse.

${ }^{21}$ Acto I. "L ucky: ... W ho from the heights of divineapathia divine athambia divine aphasia loves us dearly with some exceptions for reasons unknown".
} 
ser $D$ ios. N o lo es porque si bien desde él surge el sentido de todo lo que sucede en la obra, Godot es una fuente de sentido indolente, sin vigor ni energía, sin capacidad de emocionar y con la palabra perdida.

¿Q uién es Godot?, Godot es la modernidad moribunda que necesita del prefijo "pos". Sí, la modernidad, aquella que nació y se formó en el afán de libertad basada en criterios con carácter de verdad soportados por la razón, aquella que se desarrolló en procura de lograr la verosimilitud en función del razonamiento lógico y que dejó de nutrirse de sus fuentes para endiosarse en sí misma. Pronto, la innovación tecnológica se convirtió en lo bueno a seguir que debemos alcanzar si queremos sobrevivir en la sociedad capitalista; pronto, la autonomía se convirtió en el mejor modo de encasillar de antemano todas las posibles acciones para lograr así un mejor control cibernético de la sociedad; pronto, la modernización comenzó a concebirse como buena en sí misma, sin cuestionarse por qué modernizar; pronto, el mundo futuro será al canzado gracias a la modernización tecnológica y económica.

Por eso, todo en la obra parece moderno, sin serlo... Los personajes están esperando que algún día llegue G odot y los salve de su tedio y su sinsentido sin lograr percatarse que G odot no puede ser D ios, le está negado desde sus principios.

\section{LA TRAGEDIA POSMODERN A}

D e lo que hemos dicho podemos ya entender el carácter de tragedia de la obra. La tragedia aparece cuando junto a Pozzo, V ladimiro y el engañoso niño mensajero caemos en cuenta de la inacción de la espera en un inactivo G odot que referencia todo. Se trata no de una tragedia como la griega clásica en la que dos nociones rivales de bien contraponen acciones distintas y por no poderse hallar solución a tal contradicción amerita de la intervención de los dioses. En ello, la tragedia clásica mostraba el fundamento de lo bueno que sustentaba a la comunidad y le brindaba sentido. Pero, en Esperando a G odot la tragedia es de un tipo distinto. Como dice Simpson (1991), "si D idi y Gogo no pueden ser llamados héroes trágicos, no lo es por la imposibilidad de actuar, sino por la carencia de un universo de leyes (predecible, ordenado) en el cual actuar" (p. 84, traducción libre). Esta tragedia nos muestra no las contradicciones desde un sedimento fundamental quebrinda sentido, sino la desaparición del fundamento mismo y por lo tanto el advenimiento del sinsentido en la posmodernidad.

Ante esto quizás sólo queda la opción del suicidio. ¿Por qué?... Porque la tragedia de la espera de Godot les ha revelado el sinsentido de sus vidas, su destierro, su enajenación. La vida, ese destello de luz que brilla un instante, esen 
tanto que el sepulturero la asista en el parto. La vida es vida en tanto que una muerte le espere para volverla a la nada y finalizarla. Así, la muerte es el final, el fin, la meta que será coronada. La muerte es el fin de la vida. Y la razón de ser del hombremoderno ha desaparecido. Por ello la vida del hombre contemporáneo no puede tener epitafio sino una pequeña referencia a los datos objetivados de las fechas de nacimiento y muerte. No hay posibilidad de dar cuenta, desde la muerte, de la vida del hombre porque no hay una época que permita hacer sentido de la vida.

El suicidio parece surgir aquí como una búsqueda límite por darle fin a la vida...

0 quizás entre la tensión que surge al rededor de un Pozzo que menosprecia el tiempo cronológico y un Vladimiro quecuenta su devenir, entreun V ladimiro que en la cuenta despierta de su sueño para ver el sinsentido desu vida tragicómica y un Estragón sarcástico, entre un cambio hacia un árbol reverdecido - entre todo ello- aparezca el sentido de la vida en su ausencia y por ello quizás sea mejor esperar esperando con lo único que les es más propio, la compañía en el cuidado de V ladimiro y Estragón.

\section{AGRADECIMIENTO}

Q uisiera agradecer a los profesores Hernán López Garay y M iguel Crespo Alvarado por haber acompañado las reflexiones en torno a Esperando a G odot.

\section{REFERENCIAS}

Arendt, $\mathrm{H}$ annah [1958] 1998. The H uman Condition. Chicago: The University of Chicago Press. $2^{\text {da }}$ edición.

Beckett, Samuel [1952] 1954. Waiting for G odot. Traducción deSamuel Beckett. http:/ /samuel-beckett.net/Waiting_for_Godot_Part1.html y http://samuel-beckett.net/ Waiting_for_Godot_Part2.html Fecha de última consulta: 23/08/05

Butler, Lance St. John. 1991. "Waiting for Godot and Philosophy". En Approaches to Teaching Waiting for Godot. N ew York: Modern Language Association. June Schlueter y Enoch Brater.

Campos M ónica. s.f. El día y el instanteen los personajes de Esperando a $G$ odot. http:/ /www.filo.uba.ar/contenidos/carreras/letras/catedras/litinglesa/sitio/campos.htm Fecha de última consulta: 10/08/05

Rabinovitz, Rubin. 1977. "Time, space, and verisimilitude in Samuel Beckett'sfiction". Journal of Beckett Studies $\mathrm{N}$ ㅇ. 2. Summer.

Salatino deZubiría, M .C. 2001. "Poética deuna espera: En Attendant Godot deSamuel Beckett". Acta Literaria $\mathrm{N}$ 26. Universidad de Concepción, Chile. 
Sherzer, D ina. 1991. "Teaching Waiting for G odot, in a French D rama Course". En ApproachestoTeachingWaiting for G odot. N ew York: M odern LanguageA ssociation. June Schlueter y Enoch Brater.

Simpson, M ary Scott. 1991. "Waiting for Godot and the Forms of Tragedy". En ApproachestoTeachingWaiting for $\mathrm{G}$ odot. N ew York: M odern Language Association. June Schlueter y Enoch Brater.

Velissariou, Aspasia. 1982. "Language in Waiting for Godot". Journal of Beckett Studies $\mathrm{N} 0$ 8. Autumn. 